\title{
Exercise training and pulmonary rehabilitation: new insights and remaining
} challenges

\author{
T. Troosters*, R. Gosselink*, W. Janssens ${ }^{\#}$ and M. Decramer ${ }^{\#}$
}

ABSTRACT: Pulmonary rehabilitation is now regarded as an evidence-based treatment for symptomatic patients with chronic obstructive pulmonary disease. It has been shown to enhance exercise tolerance, improve symptoms and health-related quality of life, and reduce exacerbations in patients with recurrent exacerbations. In this article we review the mechanisms through which exercise training results in beneficial effects. We also review three challenges that currently remain: 1) the fine tuning of exercise training and multidisciplinary programmes; 2) the timing of rehabilitation; and 3) efforts to enhance the accessibility and adherence to pulmonary rehabilitation programmes. Further research is needed in order to apply the now well-established principles of pulmonary rehabilitation to unusual patient populations, or patient populations that are unlikely to participate in conventional outpatient programmes.

\section{KEYWORDS: Chronic obstructive pulmonary disease, muscle, physical activity, rehabilitation}

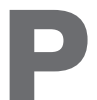

ulmonary rehabilitation is a dynamic therapeutic field which has rapidly evolved over the last decades. Exercise training remains the cornerstone of rehabilitation programmes and offers benefits to exercise tolerance that are complementary to the effects obtained from pharmacotherapy in patients with chronic obstructive pulmonary disease (COPD). Both pharmacotherapy and rehabilitation enhance health-related quality of life and exercise tolerance, and reduce exacerbation rate; however, the mechanisms leading to these benefits are different. Pharmacotherapy essentially improves lung function, pulmonary inflammation and dynamic hyperinflation during exercise, while pulmonary rehabilitation impacts on the systemic (extrapulmonary) consequences of the disease (physiological, psychological and perceived symptoms) [1]. Contemporary "combination therapy" should, therefore, include optimal pharmacotherapy and pulmonary rehabilitation programmes. This is more increasingly recognised by those to have to pay for health care. A recent example is the reimbursement of pulmonary rehabilitation by the Medicare system in the USA as of January 1, 2010.

Clearly, pulmonary rehabilitation has the most solid foundation in patients with COPD. In recent years, however, comparable effects have been shown in other respiratory diseases, including restrictive lung diseases [2], pulmonary fibrosis [3], stable pulmonary hypertension [4] and following lung transplantation $[5,6]$.

Although the effects of pulmonary rehabilitation are recognised, several challenges remain and avenues for further improvement of the field remain open. These challenges are directly related to the intervention offered, to the timing of the intervention and to the organisational aspects, the access and flexibility of programmes. These aspects will be reviewed in more detail later.

\section{THE INTERVENTION OFFERED}

Pulmonary rehabilitation programmes are multidisciplinary programmes that are built around an exercise training intervention. The aim of the exercise training is to reverse the systemic consequences of COPD, in particular the skeletal muscle dysfunction. Furthermore, exercise training enhances the mechanical efficiency of physical activities (particularly walking) and may also reduce the sensitivity to dyspnoea. In order to reverse the skeletal muscle abnormalities, exercise training needs to be of high intensity in order to result in an anabolic stimulus. When exercise training is offered at a high intensity it improves

\section{AFFILIATIONS}

*Dept of Rehabilitation Sciences and Respiratory Rehabilitation and Respiratory Division, Katholieke Universiteit Leuven, and ${ }^{\#}$ Katholieke Universiteit Leuven and University Hospital Gasthuisberg, Respiratory Division, Leuven, Belgium.

\section{CORRESPONDENCE}

T. Troosters

Respiratory Rehabilitation and Respiratory Division

UZ Gasthuisberg; Herestraat 49 B-3000 Leuven

Belgium

E-mail: Thierry.Troosters@

med.kuleuven.be

Received:

Nov 262009

Accepted after revision:

Dec 232009

PROVENANCE

Submitted article, peer reviewed.

European Respiratory Review Print ISSN 0905-9180

Online ISSN 1600-0617 
the oxidative capacity of the skeletal muscle. This leads to enhanced exercise capacity and, through its reduction of ventilation at submaximal work loads, also reduces the symptom burden in patients. Effective exercise training enhances anabolic processes in the muscle [7]. The interventions and the principles through which they lead to enhanced exercise capacity are demonstrated in figure 1.

To be effective, a training stimulus needs to be applied three times per week for a minimum of 8 weeks. The training intensity in healthy subjects is set at $55-85 \%$ of the heart rate reserve [8]. In patients with COPD, however, the exercise limitation is often the ventilatory system, which makes it complex with whole body endurance training to impose sufficient training load to the skeletal muscle. In the last few years, strategies have been developed to enhance the local load on the skeletal muscle in severely ventilatory limited patients. One strategy is that peak ventilatory capacity can be maximised using optimal bronchodilator therapy. Thus, optimal bronchodilator therapy allows enhanced training intensity and yields superior results than training performed without optimal bronchodilator therapy [9]. Another way to enhance the maximum breathing capacity of patients is the administration of gas mixtures with a density below ambient air; helium is such a gas. Breathing a gas mixture where $\mathrm{N}_{2}$ is replaced by helium acutely enhances exercise tolerance. It unloads the respiratory system allowing a higher peripheral oxygen delivery. Heliox reduces the metabolic cost of exercise and renders the peripheral muscle less fatiguable [10]. Recently, a study showed superior effects of helium breathing during exercise training. In this study [11], a mixture of helium and an increased oxygen fraction was used, which makes it difficult to dissect the effects of helium and the effect of the oxygen supplements. Besides being impractical, the cost of these mixtures is currently substantial and may render their application expensive. Unloading the respiratory muscles, thus yielding a larger oxygen delivery to peripheral muscles, can also be achieved using noninvasive mechanical ventilation (NIMV). Although

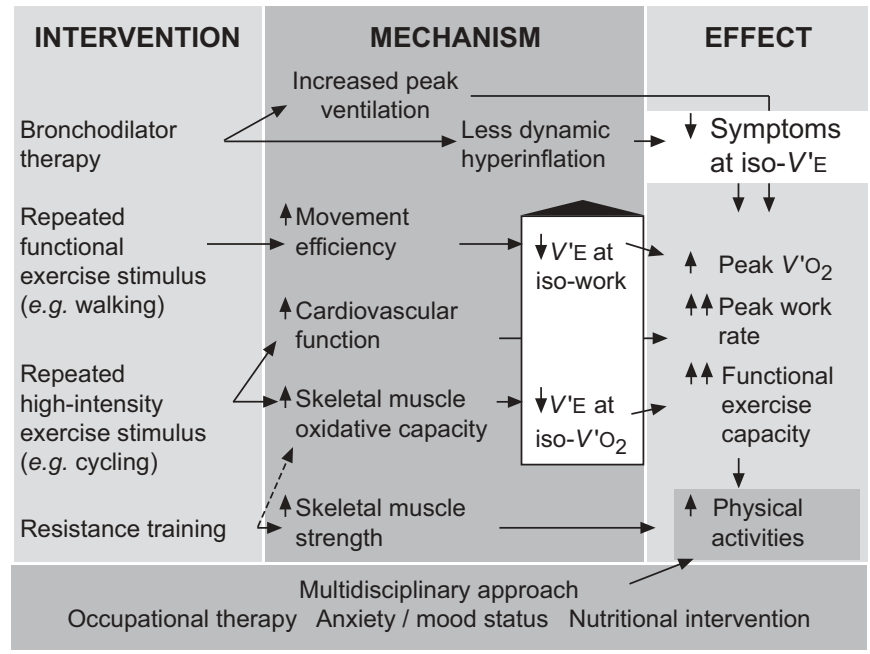

FIGURE 1. Mechanism through which pulmonary rehabilitation and pharmacotherapy may enhance physical activity. $V^{\prime} \mathrm{E}$ : minute ventilation; $V^{\prime} \mathrm{O}_{2}$ : maximal oxygen uptake. this has been performed successfully in severe COPD patients to aid the exercise regiment during pulmonary rehabilitation [12, 13], the application of NIMV during rehabilitation remains difficult and time consuming. Nevertheless, in very severe patients (e.g. those on a waiting list for lung transplantation) NIMV application may render a difficult-to-train patient trainable. In patients with respiratory failure, the combination of night-time noninvasive ventilation and daytime pulmonary rehabilitation may be more efficient [14].

The former interventions aim at supporting the impeded ventilatory system in patients with COPD. Another strategy may be to limit the ventilatory requirements during exercise. This may also give more comfort during exercise training to the ventilatory limited patient or, alternatively, allow for a higher training intensity. The first approach is to offer oxygen supplements to patients. This reduces the ventilatory drive and, thus, pulmonary ventilation for a given work load at the expense of hypercapnia. Due to the enhanced oxygen delivery it also delays lactic acidosis, which further contributes to reduced ventilator needs. EMTNER et al. [15] showed that it was possible to train patients at a higher training intensity when oxygen supplements were given. As a result they showed larger improvements in whole body endurance in the patients who trained with oxygen supplements [15]. A second approach may be to reduce the muscle bulk used during the training; this obviously reduces the metabolic demand of exercise. If training load is focused on a smaller group of muscles these can be loaded more without utilising the full ventilator capacity. DolmaGE et al. [16] used this paradigm in a study comparing single leg cycling exercise training to conventional cycling training and showed superior effects when each leg was trained separately. A final approach to reduce the ventilator needs during exercise is to use interval training rather than endurance training. During interval training small bursts of training are offered to patients. Due to the short exercise duration and the slow kinetics of pulmonary ventilation, the ventilation does not rise to high levels, even when the imposed work load is high. Interval training has comparable effects to endurance training [17] and leads to skeletal muscle hypertrophy [18]. Importantly patients reported fewer symptoms during this training regimen and less unexpected stops were observed.

The final strategy of interventions is specifically aimed at enhancing skeletal muscle force. Specific resistance training can be applied to enhance peripheral muscle function and, although some cross-over effect towards enhanced whole body exercise tolerance may be expected, the effect is particularly seen in the enhanced peripheral muscle strength and endurance. These are clearly important features to daily life activities. Specific resistance training has been applied in several studies and summarised in several excellent review papers [19, 20]. Resistance training (or weight lifting) significantly enhances skeletal muscle strength when applied alone or in combination with whole body exercise training [21]. Therefore, it is a powerful rehabilitation tool to enhance the skeletal muscle "machinery". More recently, several authors have used "nonphysiological" neuromuscular electrical stimulation to enhance peripheral muscle function [22]. Electrical stimulation has also been shown to be effective in enhancing skeletal muscle function. However, as the stimulations are not 
physiologically evoked and are not embedded in neuromuscular coupling pathways, we would advise restricting the use of neuromuscular electrical stimulation to situations where prevention of the loss of skeletal muscle function is required, or in programmes where neuromuscular electrical stimulation is combined with conventional pulmonary rehabilitation. The effects of neuromuscular electrical stimulation and resistance training may be amplified by the addition of pharmacotherapy directed to improve skeletal muscle function. Testosterone supplements in addition to resistance training have been proven in hypogonadal males to restore skeletal muscle strength more rapidly compared with resistance training alone [23]. Other targets for skeletal muscle directed pharmacotherapy are being identified but, to date, its clinical application has not been validated [24]. Such therapy can target scavenging processes of oxidative stress in susceptible patients [25], anabolic signalling and substrates or key proteins of the muscle machinery. Patient selection for supplemental interventions will be crucial to show their effectiveness.

All the above interventions focus on fine-tuning the exercise training intervention. Pulmonary rehabilitation, however, consists of more than just exercise training. Surprisingly, little research has been presented in recent years on the specific effects of introducing other disciplines involved in pulmonary rehabilitation. In particular, there is little knowledge on how to transfer the gains in exercise capacity into enhanced participation in daily life activities. Several studies have assessed the effects of rehabilitation programmes on physical activity. The improvement of physical activity levels is not guaranteed after pulmonary rehabilitation, as is indicated in table 1 and figure 2 . Importantly, it is unknown how much improvement in physical activity is a clinically relevant improvement. Any increase in the proportion of patients meeting the required amount of physical activity for healthy ageing would be a significant benefit for both patients and society. Whether pulmonary rehabilitation enhances the proportion of patients meeting these goals (30 min of physical activity at moderate intensity on at least 5 days $^{*}$ week $^{-1}$ ) [35] has not yet been investigated. Indeed, surprisingly little research has focused on ways to facilitate the transfer of benefits to physical activity. A study from the Netherlands suggested that the use of simple pedometer devices may provide patients with real-time feedback on their physical activity levels [34]. When appropriate guidance is given this may enhance physical activity levels, much like in healthy subjects [36]. Whether other behavioural interventions may help to achieve a long-term change towards a more physically active lifestyle remains to be investigated in COPD.

\section{TIMING OF THE REHABILITATION INTERVENTION}

Most guidelines report extensively on the benefits of pulmonary rehabilitation in patients with stable lung disease. Much less is known about the effects of pulmonary rehabilitation immediately after or even during acute exacerbations. A recent meta-analysis summarised the currently available evidence and concluded that pulmonary rehabilitation established after exacerbations enhances health-related quality of life, improves exercise capacity and reduces the risk for re-admissions [37]. Following severe exacerbations leading to hospital admission, patients are at risk for re-admission and survival is severely reduced. Acutely reduced skeletal muscle force [38] and depressive symptoms (identified as an independent risk factor for mortality after exacerbations) [39] are surely amenable to rehabilitation therapy. Furthermore, patients are extremely inactive after acute exacerbations and it is very infrequent to observe recovery to normal physical activity levels in the

TABLE 1 Changes in physical activity after pulmonary rehabilitation

\begin{tabular}{|c|c|c|c|c|c|}
\hline First author [Ref.] & Patients & $\begin{array}{l}\text { Sessions of } \\
\text { supervised } \\
\text { rehabilitation }\end{array}$ & $\begin{array}{c}\text { Duration of } \\
\text { rehabilitation } \\
\text { weeks }\end{array}$ & Instrument to measure physical activity & Outcome \\
\hline Steele $[26]$ & 38 & 16 & 8 & TA: Tritrac R3D accelerometer & VMU per min \\
\hline Steele $[27]$ & 111 & 16 & 8 & TA: RT3 accelerometer & VMU \\
\hline CoRonado [28] & 15 & 36 & 3 & UA: self-contained activity monitor & Time at low + medium act $\%$ \\
\hline Standard & 59 & 14 & 7 & UA: ambulatory activity monitor & Counts \\
\hline Individualised & 64 & 14 & 7 & UA: Z80-32k V1 Int & Counts \\
\hline \multicolumn{6}{|l|}{ PITTA [31] } \\
\hline 3 months & 29 & 36 & 12 & TA: dynaport & Walking time \\
\hline 6 months & 29 & 60 & 24 & TA & Walking time \\
\hline WALKER [32] & 23 & 16 & 8 & UA: actiwatch & Time spent mobile \% \\
\hline Classic & 8 & NR & 9 & PM: Yamax digi walker & Steps \\
\hline Classic+pedom & 8 & NR & 9 & PM: Yamax digi walker & Steps \\
\hline
\end{tabular}

Data are presented as n. pedom: pedometer feedback; TA: triaxial accelerometer; UA: uniaxial accelerometer; PE: pedometer (electronic); PM: pedometer (mechanical); NR: not reported; VMU: vector magnitude units. Please also refer to figure 2. 


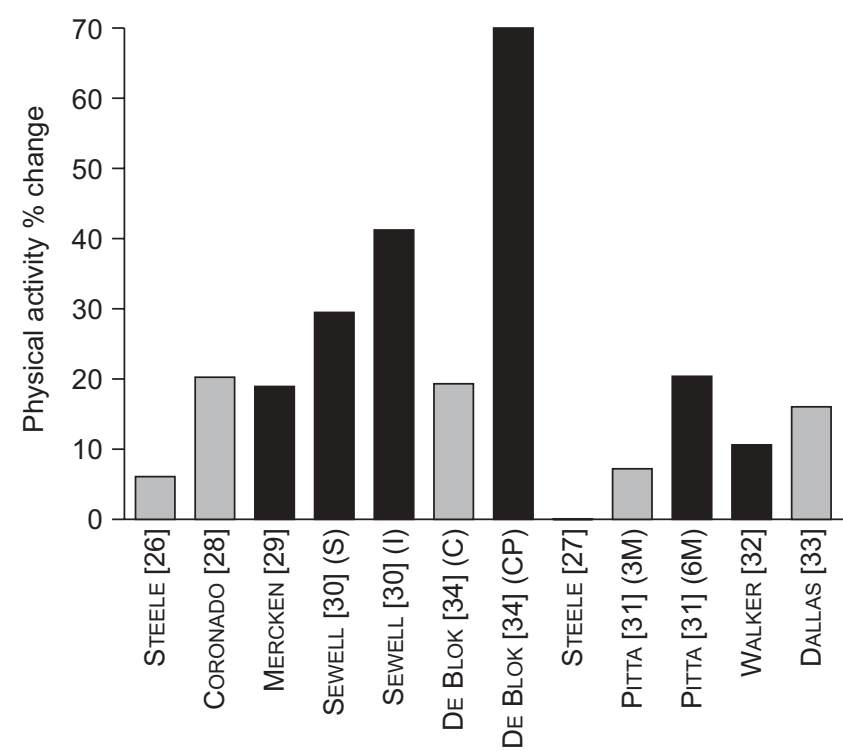

First author [Ref.]

FIGURE 2. Improvement of physical activity. studies that were statistically significant; 1 : studies that were statistically nonsignificant. S: standard; I: individualised; C: classic; CP: classic+pedometer feedback; 3M: 3 months; $6 \mathrm{M}$ : 6 months.

month following a hospital admission [40]. Obviously rehabilitation faces many challenges in the period immediately after an exacerbation. Adherence to the intervention and recruitment of patients is extremely difficult [41]. In addition, exercises need to be adapted to the ventilatory impairment of the patient. Patients need to be encouraged to become physically active after exacerbations, which may warrant input from occupational therapists and/or psychological counselling to deal with anxiety or depression. Furthermore, interventions enhancing self-management are useful in these frail patients [42].

On the other side of the disease spectrum, in patients with milder disease, the efficacy of pulmonary rehabilitation is not yet supported by substantial evidence. A recent trial investigating the impact of pulmonary rehabilitation in the community in patients with a forced expiratory volume in $1 \mathrm{~s}$ of $\sim 60 \%$ of the predicted value showed only modest effects [43]. Others studies have shown clear effects in these patients [44]. Further studies are required to investigate the potential of rehabilitation early in the disease process to prevent the systemic consequences of COPD. Such studies need long-term followup and large study populations, as the main effect of such programmes is the prevention of the incidence of systemic consequences, rather than their cure. In addition, such studies can investigate the role of enhanced physical activity on lung function decline [45].

\section{ACCESS AND FLEXIBILITY}

One of the most important challenges of pulmonary rehabilitation is retention in the programme and referral to programmes. A recent Danish study showed that only $60 \%$ of patients visiting an outpatient university hospital clinic were offered pulmonary rehabilitation [46]. In Scotland (UK) only $23 \%$ of primary-care practitioners were able to refer their patients to a pulmonary rehabilitation programme [47]. In Canada only $9 \%$ of patients with COPD were referred for pulmonary rehabilitation [48]. A survey conducted in the UK in 2001 suggests that only $1 \%$ of COPD patients receive rehabilitation [49]. Attempts should be made to make pulmonary rehabilitation available to more patients and also to keep patients in programmes once they are enrolled. Dropout from programmes and nonadherence impede on the effects of rehabilitation. A prospective study in the Netherlands identified that $23 \%$ of patients recruited in a rehabilitation programmes quit the programme prematurely. Discontinuation was often due to medical reasons (exacerbations and infarction). Patients with poor attendance were more likely to have less social support, tended to be smokers and had a lower fat-free mass. A study from the UK reported a $31 \%$ dropout rate and identified low muscle force, smoking history, poor health-related quality of life and depressive mood status as predictors for dropout [50]. It appears as if patients not completing the rehabilitation programme are in fact theoretically good candidates for rehabilitation. Several modifications could be made to programmes to better fit with the patients' abilities to cope with the programmes. In the future, telemedicine could perhaps help to further individualise exercise training programmes for patients. However, care should be taken not to create easily accessible programmes in the community or at home at the expense of the effectiveness of the programmes. Recently a number of large studies [43, 51, 52] suggested that it is challenging to reproduce the effects of pulmonary rehabilitation observed in single-centre studies, and in multi-centre trials, where perhaps less expertise is available to individualise programmes. However, one study suggests otherwise [53]; close supervision of patients by an expert team appears to be a factor associated with the overall success, and particularly the physiological benefits, of programmes [54].

\section{CONCLUSIONS}

Pulmonary rehabilitation is now recognised an effective treatment that should be integrated in the management of symptomatic patients with COPD. Three important challenges lie ahead: 1) the implementation of these programmes into the lines of healthcare so that they are accessible to most patients who would benefit; 2) ensuring the quality of these programmes so that the effects obtained in single-centre studies and carried out in centres of excellence can be applied in less specialised centres; and 3) facilitating the transfer of the obtained rehabilitation benefits into long lasting improvements in the behaviour of patients (including smoking cessation and physical activity)

Pulmonary rehabilitation programmes are built around highintensity exercise training to enhance exercise tolerance, skeletal muscle function and functional exercise capacity. In individual patients, other healthcare professionals, including physiotherapists, psychologists, nutritional specialists, occupational therapist, nurse specialists and doctors, contribute to the full effects of rehabilitation on health-related quality of life, psychological morbidity, reduced exacerbation rate and enhanced participation in daily life (physical activity). The latter remains a challenge and further research should focus on improving the effectiveness of rehabilitation programmes on enhancing physical activity, which is the cornerstone to long lasting benefits. 
This may be of particular importance following exacerbations when physical activity levels drop dramatically and continued inactivity is a predictor of successive events.

It is important to recognise the potential of pulmonary rehabilitation in clinical practice. We have now entered the era of fine tuning the intervention and making it available, in an effective format, to larger groups of patients. We realise that this may require efforts from scientists, healthcare providers, policy makers and patients. Given the proven effectiveness of these interventions, however, no effort should be spared.

\section{SUPPORT STATEMENT}

This article was supported by grant G.0598.09N from the Fonds Wetenschappelijk Onderzoek (Brussels, Belgium).

\section{STATEMENT OF INTEREST}

None declared.

\section{REFERENCES}

1 Nici L, Donner C, Wouters E. American Thoracic Society/ European Respiratory Society statement on pulmonary rehabilitation. Am J Respir Crit Care Med 2006; 173: 1390-1413.

2 Salhi B, Troosters T, Behaegel M. Effects of pulmonary rehabilitation in patients with restrictive lung diseases. Chest 2009; [Epub ahead of print DOI: 10.1378/chest.09-0241].

3 Holland AE, McDonald CF. Pulmonary rehabilitation and interstitial lung disease. Thorax 2009; 64: 548.

4 de Man FS, Handoko ML, Groepenhoff H. Effects of exercise training in patients with idiopathic pulmonary arterial hypertension. Eur Respir J 2009; 34: 669-675.

5 Munro PE, Holland AE, Bailey M. Pulmonary rehabilitation following lung transplantation. Transplant Proc 2009; 41: 292-295.

6 Maury G, Langer D, Verleden G. Skeletal muscle force and functional exercise tolerance before and after lung transplantation: a cohort study. Am J Transplant 2008; 8: 1275-1281.

7 Vogiatzis I, Stratakos G, Simoes DC. Effects of rehabilitative exercise on peripheral muscle TNF $\alpha$, IL-6, IGF-I and MyoD expression in patients with COPD. Thorax 2007; 62: 950-956.

8 American College of Sports Medicine Position Stand. The recommended quantity and quality of exercise for developing and maintaining cardiorespiratory and muscular fitness, and flexibility in healthy adults. Med Sci Sports Exerc 1998; 30: 975-991.

9 Casaburi R, Kukafka D, Cooper CB. Improvement in exercise tolerance with the combination of tiotropium and pulmonary rehabilitation in patients with COPD. Chest 2005; 127: 809-817.

10 Chiappa GR, Queiroga F Jr, Meda E. Heliox improves oxygen delivery and utilization during dynamic exercise in patients with chronic obstructive pulmonary disease. Am J Respir Crit Care Med 2009; 179: 1004-1010.

11 Eves ND, Sandmeyer LC, Wong EY. Helium-hyperoxia: a novel intervention to improve the benefits of pulmonary rehabilitation for patients with COPD. Chest 2009; 135: 609-618.

12 van't Hul A, Gosselink R, Hollander P. Training with inspiratory pressure support in patients with severe COPD. Eur Respir J 2006; 27: 65-72.

13 Hawkins P, Johnson LC, Nikoletou D. Proportional assist ventilation as an aid to exercise training in severe chronic obstructive pulmonary disease. Thorax 2002; 57: 853-859.

14 Duiverman ML, Wempe JB, Bladder G. Nocturnal non-invasive ventilation in addition to rehabilitation in hypercapnic patients with COPD. Thorax 2008; 63: 1052-1057.
15 Emtner M, Porszasz J, Burns M. Benefits of supplemental oxygen in exercise training in non-hypoxemic COPD patients. Am J Respir Crit Care Med 2003; 168: 1034-1042.

16 Dolmage TE, Goldstein RS. Effects of one-legged exercise training of patients with COPD. Chest 2008; 133: 370-376.

17 Puhan MA, Busching G, Schunemann HJ. Interval versus continuous high-intensity exercise in chronic obstructive pulmonary disease: a randomized trial. Ann Intern Med 2006; 145: 816-825.

18 Vogiatzis I, Terzis G, Nanas S. Skeletal muscle adaptations to interval training in patients with advanced COPD. Chest 2005; 128 3838-3845.

19 O'Shea SD, Taylor NF, Paratz J. Peripheral muscle strength training in COPD: a systematic review. Chest 2004; 126: 903-914.

20 Houchen L, Steiner MC, Singh SJ. How sustainable is strength training in chronic obstructive pulmonary disease? Physiotherapy 2009; 95: 1-7.

21 Ortega F, Toral J, Cejudo P. Comparison of effects of strength and endurance training in patients with chronic obstructive pulmonary disease. Am J Respir Crit Care Med 2002; 166: 669-674.

22 Sillen MJ, Speksnijder CM, Eterman RM. Effects of neuromuscular electrical stimulation of muscles of ambulation in patients with chronic heart failure or COPD: a systematic review of the Englishlanguage literature. Chest 2009; 136: 44-61.

23 Casaburi R, Bhasin S, Cosentino L. Anabolic effects of testosterone replacement and strength training in men with COPD. Am J Respir Crit Care Med 2004; 170: 870-878.

24 Goodyear LJ. The exercise pill - too good to be true? N Engl J Med 2008; 359: 1842-1844.

25 Rabinovich RA, Ardite E, Mayer AM. Training depletes muscle glutathione in patients with chronic obstructive pulmonary disease and low body mass index. Respiration 2006; 73: 757-761.

26 Steele BG, Belza B, Hunziker J. Monitoring daily activity during pulmonary rehabilitation using a triaxial accelerometer. $J$ Cardiopulm Rehabil 2003; 23: 139-142.

27 Steele BG, Belza B, Cain KC. A randomized clinical trial of an activity and exercise adherence intervention in chronic pulmonary disease. Arch Phys Med Rehabil 2008; 89: 404-412.

28 Coronado M, Janssens JP, de Muralt B. Walking activity measured by accelerometry during respiratory rehabilitation. J Cardiopulm Rehabil 2003; 23: 357-364.

29 Mercken EM, Hageman GJ, Schols AM. Rehabilitation decreases exercise-induced oxidative stress in chronic obstructive pulmonary disease. Am J Respir Crit Care Med 2005; 172: 994-1001.

30 Sewell L, Singh SJ, Williams JE. Can individualized rehabilitation improve functional independence in elderly patients with COPD? Chest 2005; 128: 1194-1200.

31 Pitta F, Troosters T, Probst V. Are patients with COPD more active after pulmonary rehabilitation. Chest 2008; 134: 273-280.

32 Walker PP, Burnett A, Flavahan PW. Lower limb activity and its determinants in COPD. Thorax 2008; 63: 683-689.

33 Dallas MI, McCusker C, Haggerty MC. Using pedometers to monitor walking activity in outcome assessment for pulmonary rehabilitation. Chron Respir Dis 2009; 6: 217-224.

34 de Blok BM, de Greef MH, ten Hacken NH. The effects of a lifestyle physical activity counseling program with feedback of a pedometer during pulmonary rehabilitation in patients with COPD: a pilot study. Patient Educ Couns 2006; 61: 48-55.

35 Haskell WL, Lee IM, Pate RR. Physical activity and public health. Updated recommendation for adults from the American College of Sports Medicine and the American Heart Association. Circulation 2007; 116: 1081-1093.

36 Bravata DM, Smith-Spangler C, Sundaram V. Using pedometers to increase physical activity and improve health: a systematic review. JAMA 2007; 298: 2296-2304.

37 Puhan M, Scharplatz M, Troosters T. Pulmonary rehabilitation following exacerbations of chronic obstructive pulmonary disease. Cochrane Database Syst Rev 2009; CD005305. 
38 Spruit M, Gosselink R, Troosters T. Muscle force during an acute exacerbation in hospitalised COPD patients and its relationship with CXCL8 and IGF-1. Thorax 2003; 58: 752-756.

39 Yohannes AM, Baldwin RC, Connolly MJ. Predictors of 1-year mortality in patients discharged from hospital following acute exacerbation of chronic obstructive pulmonary disease. Age Ageing 2005; 34: 491-496.

40 Pitta F, Troosters T, Probst VS. Physical activity and hospitalization for exacerbation of COPD. Chest 2006; 129: 536-544.

41 Eaton T, Young P, Fergusson W. Does early pulmonary rehabilitation reduce acute health-care utilization in COPD patients admitted with an exacerbation? A randomized controlled study. Respirology 2009; 14: 230-238.

42 Gadoury MA, Schwartzman K, Rouleau M. Self-management reduces both short- and long-term hospitalisation in COPD. Eur Respir J 2005; 26: 853-857.

43 van Wetering CR, Hoogendoorn M, Mol SM. Short- and long-term efficacy of a community-based COPD management program in less advanced COPD: a randomized controlled trial. Thorax 2009; [Epub ahead of print DOI: 10.1136/thx.2009.118620].

44 Casaburi R, Patessio A, Ioli F. Reductions in exercise lactic acidosis and ventilation as a result of exercise training in patients with obstructive lung disease. Am Rev Respir Dis 1991; 143: 9-18.

45 Stav D, Raz M, Shpirer I. Three years of pulmonary rehabilitation: inhibit the decline in airflow obstruction, improves exercise endurance time, and body-mass index, in chronic obstructive pulmonary disease. BMC Pulm Med 2009; 9: 26.
46 Lange $\mathrm{P}$, Andersen KK, Munch E. Quality of COPD care in hospital outpatient clinics in Denmark: The KOLIBRI study. Respir Med 2009; 103: 1657-1662.

47 Cleland J, Mackenzie M, Small I. Management of COPD in primary care in north-east Scotland. Scott Med J 2006; 51: 10-14.

48 Bourbeau J, Sebaldt RJ, Day A. Practice patterns in the management of chronic obstructive pulmonary disease in primary practice: the CAGE study. Can Respir J 2008; 15: 13-19.

49 Yohannes AM, Connolly MJ. Pulmonary rehabilitation programmes in the UK: a national representative survey. Clin Rehabil 2004; 18: 444-449.

50 Garrod R, Marshall J, Barley E. Predictors of success and failure in pulmonary rehabilitation. Eur Respir J 2006; 27: 788-794.

51 Maltais F, Bourbeau J, Shapiro S. Effects of home-based pulmonary rehabilitation in patients with chronic obstructive pulmonary disease: a randomized trial. Ann Intern Med 2008; 149: 869-878.

52 Effing T. Self management in patients with COPD, The COPE-II study. PhD thesis. Radboud Universiteit, Nijmegen, the Netherlands. 2009.

53 Guell MR, de LP, Galdiz JB. [Home vs hospital-based pulmonary rehabilitation for patients with chronic obstructive pulmonary disease: a Spanish multicenter trial]. Arch Bronconeumol 2008; 44: 512-518.

54 Puente-Maestu L, Sanz ML, Sanz P. Comparison of effects of supervised versus self-monitored training programmes in patients with chronic obstructive pulmonary disease. Eur Respir J 2000; 15: 517-525. 